\title{
The comprehensive summary of surgical versus non-surgical treatment for obesity: a systematic review and meta-analysis of randomized controlled trials
}

\author{
Ji Cheng ${ }^{1}$, Jinbo Gao ${ }^{1}$, Xiaoming Shuai ${ }^{1}$, Guobin Wang ${ }^{1}$ and Kaixiong Tao ${ }^{1}$ \\ 1 Department of Gastrointestinal Surgery, Union Hospital, Tongji Medical College, Huazhong University of Science and \\ Technology, Wuhan, Hubei, China \\ Correspondence to: Kaixiong Tao, email: kaixiongtaowhuh@126.com \\ Keywords: obesity; bariatric surgery; meta-analysis; systematic review; Pathology Section \\ Received: April 16, $2016 \quad$ Accepted: May 20, $2016 \quad$ Published: May 24, 2016
}

ABSTRACT

Background: Bariatric surgery has emerged as a competitive strategy for obese patients. However, its comparative efficacy against non-surgical treatments remains ill-defined, especially among nonseverely obese crowds. Therefore, we implemented a systematic review and meta-analysis in order for an academic addition to current literatures.

Methods: Literatures were retrieved from databases of PubMed, Web of Science, EMBASE and Cochrane Library. Randomized trials comparing surgical with nonsurgical therapies for obesity were included. A Revised Jadad's Scale and Risk of Bias Summary were employed for methodological assessment. Subgroups analysis, sensitivity analysis and publication bias assessment were respectively performed in order to find out the source of heterogeneity, detect the outcome stability and potential publication bias.

Results: 25 randomized trials were eligibly included, totally comprising of 1194 participants. Both groups displayed well comparability concerning baseline parameters $(P>0.05)$. The pooled results of primary endpoints (weight loss and diabetic remission) revealed a significant advantage among surgical patients rather than those receiving non-surgical treatments $(P<0.05)$. Furthermore, except for certain cardiovascular indicators, bariatric surgery was superior to conventional arms in terms of metabolic secondary parameters $(P<0.05)$. Additionally, the pooled outcomes were confirmed to be stable by sensitivity analysis. Although Egger's test $(P<0.01)$ and Begg's test $(P<0.05)$ had reported the presence of publication bias among included studies, "Trim-and-Fill" method verified that the pooled outcomes remained stable.

Conclusion: Bariatric surgery is a better therapeutic option for weight loss, irrespective of follow-up duration, surgical techniques and obesity levels.

\section{INTRODUCTION}

Emerging as a costly burden of global healthcare system, obesity has currently attracted worldwide attentions due to its uncontrollably rising incidence, especially in industrialized countries [1]. Economically, the annual expense directly linked to overweight (body mass index 25-30) and obesity (body mass index > 30) is estimated to be almost 16 billion pounds globally
[2]. During the past three decades, the epidemiological prevalence of obesity has quadrupled to $25 \%$ among UK population, including $2.4 \%$ of which are affected by morbid obesity (body mass index $>40$ ) [3]. At present, it is appraised that approximately two thirds of overall population in US have been clinically diagnosed as overweight or obese [4]. Characterized by excessive adipose storage, obesity is commonly accompanied by a variety of comorbidities, mainly comprising of type 
2 diabetes mellitus, hypertension and cardiovascular accidents. As an integrative product of multifactorial impacts, a definite etiological explanation of obesity remains obscure, leading to the absence of effective strategies targeting its development [5].

According to SAGES [6] and NICE [7] guidelines, multicomponent interventions are currently the treatment of choice, including lifestyle intervention, dietary restriction, pharmaceutical and surgical management. Despite of its first-line status, non-surgical treatments lead to poor compliance and unfavorable endpoint satisfaction among obese patients. As is reported, the glycemic control of obesity-related diabetes is merely accomplished amid $40 \%$ of patients undergoing conventional medications [8]. Meanwhile, lifestyle reformation fails to reduce the probability of obesity-associated lethality as well as the hazards of cardiovascular accidents among obese crowds [9].

Bariatric surgery, initially reported in 1995 [10], has been regarded as an effective supplement to current regimen, especially for those suffering from severe obesity (body mass index $>40$ ) $[6,7]$. By mechanically altering the physiological mode of gastrointestinal absorption, bariatric surgery triggers remarkable decline on excessive weight, hyperglycemia, cardiovascular risk and correlative mortality compared to conservative therapeutics [4, 11, 12]. Moreover, by meta-analyzing short-term observational studies, Muller et al [8] has investigated that in contrast to non-surgical remedies, bariatric surgery favorably produces therapeutic benefit among patients with nonsevere obesity (body mass index 30-35), which is a vital addition to current guidelines that surgical intervention is recommended for patients with body mass index $>35$, especially the morbidly obese sufferers (body mass index $>40$ ) [6]. Therefore, the updated NICE guidance (CG189) has broadened the indications that as a second-line option, all patients with body mass index $>$ 30 should be assessed for bariatric surgery following the refractoriness to non-surgical interventions [2].

Nevertheless, long-term (3-year or more) efficacy of surgical versus non-surgical interventions is rarely described, especially lacking of a well summarized evidence. Additionally, the clinical value of bariatric surgery for nonseverely obese patients requires further analysis. Hence we performed this systematic review and meta-analysis in order to comprehensively make comparisons between both strategies, aiming to provide novel evidences for future guidelines.

\section{RESULTS}

\section{General characteristics}

The preliminary 1076 entries were rigorously screened to generate 25 eligible studies for pooling analysis, with a total amount of 1194 participants and individually ranging from 16 to 150 (Figure 1A). Merely 2 studies were performed by developing nations while 14 trials originated from industrialized countries, each of Australia and US accounting for the maximum amount of 5. None of the included trials featured adolescent subjects except for O'Brien 2010. Female patients dominated the sexuality proportion towards male counterparts, with a ratio of 740 to 454 . Among the included trials, in general, it was mutually comparable between both comparative interventions regarding the baseline confounding elements (Table 1).

\section{Summary of methodological assessment}

According to Revised Jadad's Scale, 13 trials were appraised as high-quality in methodology, while DSS, Heindorff 1997 and Mingrone 2002 were identified as low-quality investigations (Table 2).

Since a relatively small fraction of subitems were assessed with low risk of bias, DSS, Heindorff 1997, Mingrone 2002 and O'Brien 2013 were considered with high risk of internal bias. The overall amount of low risk subitems was 61 , followed by 37 of unclear risk and 14 of high risk, implying a substantially low risk of bias within our pooled analysis (Figure 1B).

\section{Primary endpoints}

\section{Weight loss}

\section{Overall patients}

undergoing surgical interventions had more weight loss than those receiving non-surgical managements $(P<$ 0.00001) (Figure 2).

\section{Follow-up duration}

In terms of weight loss, surgical approach was superior to conventional strategies among patients with 1-year $(P<0.00001)$, 2-year $(P<0.00001)$ or longterm (3 years or more) follow-up duration $(P<0.00001)$ (Figure 2).

\section{Surgical techniques}

Non-surgical therapeutics were unable to outstrip bariatric surgery regarding weight loss among obese patients, irrespective of sleeve gastrectomy $(P<$ 
Table 1: Baseline features of included studies

\begin{tabular}{|c|c|c|c|c|c|c|c|c|c|c|c|c|}
\hline Trial title & Country & $\begin{array}{c}\text { Trial } \\
\text { registration }\end{array}$ & Group & Sample size & Age (years) & $\begin{array}{c}\text { Sexuality } \\
(\mathbf{M} / \mathbf{F})\end{array}$ & $\begin{array}{c}\text { BMI } \\
\left(\mathrm{kg} / \mathrm{m}^{2}\right)\end{array}$ & Weight (kg) & $\begin{array}{c}\text { Waist } \\
\text { circumference } \\
(\mathrm{cm}) \\
\end{array}$ & Caucasian & Hypertension & $\begin{array}{c}\text { T2DM } \\
\text { (Prevalence/Dur } \\
\text { ation) } \\
\end{array}$ \\
\hline \multirow{2}{*}{$\begin{array}{c}\text { DIBASY[13, } \\
14]\end{array}$} & \multirow{2}{*}{ Italy } & \multirow{2}{*}{$\begin{array}{c}\text { NCT00888 } \\
836 \\
\end{array}$} & Surgery & 40 & $43.3 \pm 7.7$ & $18 / 22$ & $45.0 \pm 6.5$ & $133.8 \pm 26.7$ & $127.9 \pm 18.2$ & NA & NA & $100.0 \% / 6.0 \mathrm{y}$ \\
\hline & & & Control & 20 & $43.5 \pm 7.3$ & $10 / 10$ & $45.6 \pm 6.2$ & $136.4 \pm 21.9$ & $126.9 \pm 14.7$ & NA & NA & $100.0 \% / 6.1 y$ \\
\hline \multirow{2}{*}{$\begin{array}{c}\text { Dixon } \\
2008[15]\end{array}$} & \multirow{2}{*}{ Australia } & \multirow{2}{*}{$\begin{array}{c}\text { ACTRN12 } \\
605000159 \\
651 \\
\end{array}$} & Surgery & 30 & $46.6 \pm 7.4$ & $15 / 15$ & $37.0 \pm 2.7$ & $105.6 \pm 13.8$ & $114.1 \pm 10.2$ & NA & $28(93.3 \%)$ & $100.0 \% /<2 y$ \\
\hline & & & Control & 30 & $47.1 \pm 8.7$ & $13 / 17$ & $37.2 \pm 2.5$ & $105.9 \pm 14.2$ & $116.0 \pm 10.0$ & NA & $27(90.0 \%)$ & $100.0 \% /<2 y$ \\
\hline \multirow{2}{*}{$\begin{array}{c}\text { Dixon } \\
2012[16]\end{array}$} & \multirow{2}{*}{ Australia } & \multirow{2}{*}{$\begin{array}{c}\text { ACTRN12 } \\
605000161 \\
628 \\
\end{array}$} & Surgery & 30 & $47.4 \pm 8.8$ & $17 / 13$ & $46.3 \pm 6.0$ & $134.9 \pm 22.1$ & $136.1 \pm 13.1$ & NA & $15(50.0 \%)$ & $33.3 \% / \mathrm{NA}$ \\
\hline & & & Control & 30 & $50.0 \pm 8.2$ & $18 / 12$ & $43.8 \pm 4.9$ & $126.0 \pm 19.3$ & $126.6 \pm 13.1$ & NA & $17(56.7 \%)$ & $33.3 \% / \mathrm{NA}$ \\
\hline \multirow{2}{*}{$\operatorname{DSS}[17,18]$} & \multirow{2}{*}{ USA } & \multirow{2}{*}{$\begin{array}{c}\text { NCT00641 } \\
251 \\
\end{array}$} & Surgery & 60 & $49.0 \pm 9.7$ & $22 / 38$ & $34.9 \pm 2.9$ & $98.8 \pm 13.2$ & $114.0 \pm 9.7$ & $33(55.0 \%)$ & NA & $100.0 \% / 8.9 y$ \\
\hline & & & Control & 60 & $49.0 \pm 7.7$ & $26 / 34$ & $34.3 \pm 3.1$ & $97.9 \pm 16.3$ & $113.0 \pm 11.6$ & $30(50.0 \%)$ & NA & $100.0 \% / 9.1 \mathrm{y}$ \\
\hline \multirow{2}{*}{$\begin{array}{c}\text { Heindorff } \\
1997[19]\end{array}$} & \multirow{2}{*}{ Denmark } & \multirow{2}{*}{ NA } & Surgery & 8 & $22.0-41.0$ & $2 / 6$ & $43.0-54.0$ & NA & NA & NA & NA & NA/NA \\
\hline & & & Control & 8 & $21.0-43.0$ & $7 / 1$ & $40.0-56.0$ & NA & NA & NA & NA & NA/NA \\
\hline \multirow{2}{*}{$\begin{array}{c}\text { Liang } \\
2013[20]\end{array}$} & \multirow{2}{*}{ China } & \multirow{2}{*}{$\begin{array}{c}\text { NCT01435 } \\
980\end{array}$} & Surgery & 31 & $50.8 \pm 5.4$ & $22 / 9$ & $30.5 \pm 0.9$ & $82.0 \pm 3.5$ & NA & $0(0.0 \%)$ & $31(100.0 \%)$ & $100.0 \% / 7.4 \mathrm{y}$ \\
\hline & & & Control & 70 & $51.4 \pm 6.3$ & $48 / 22$ & $30.3 \pm 1.7$ & $81.5 \pm 4.4$ & NA & $0(0.0 \%)$ & $70(100.0 \%)$ & $100.0 \% / 7.2 y$ \\
\hline \multirow{2}{*}{$\begin{array}{l}\text { Mingrone } \\
2002[21]\end{array}$} & \multirow{2}{*}{ Italy } & \multirow{2}{*}{ NA } & Surgery & 46 & $30.0-45.0$ & $7 / 39$ & $48.2 \pm 5.0$ & $133.9 \pm 16.2$ & NA & NA & $0(0.0 \%)$ & $0.0 \% / 0.0 \mathrm{y}$ \\
\hline & & & Control & 33 & $30.0-45.0$ & $4 / 29$ & $48.2 \pm 7.7$ & $130.9 \pm 24.5$ & NA & NA & $0(0.0 \%)$ & $0.0 \% / 0.0 \mathrm{y}$ \\
\hline \multirow{2}{*}{$\begin{array}{l}\text { O'Brien } \\
2006[22]\end{array}$} & \multirow{2}{*}{ Australia } & \multirow{2}{*}{\begin{tabular}{|c|} 
ACTRN12 \\
605000113 \\
651 \\
\end{tabular}} & Surgery & 40 & $41.8 \pm 6.4$ & $10 / 30$ & $33.7 \pm 1.8$ & $96.1 \pm 11.2$ & $103.3 \pm 10.0$ & NA & $9(22.5 \%)$ & $\mathrm{NA} / \mathrm{NA}$ \\
\hline & & & Control & 40 & $40.7 \pm 7.0$ & $9 / 31$ & $33.5 \pm 1.4$ & $93.6 \pm 11.9$ & $99.4 \pm 9.4$ & NA & $7(17.5 \%)$ & NA/NA \\
\hline \multirow{3}{*}{$\begin{array}{c}\text { O'Brien } \\
2010[23] \\
\end{array}$} & \multirow{2}{*}{ Australia } & ACTRN12 & Surgery & 25 & $16.5 \pm 1.4$ & $9 / 16$ & $42.3 \pm 6.1$ & $120.7 \pm 25.3$ & $120.8 \pm 14.2$ & NA & NA & NA/NA \\
\hline & & 605000160 & Control & 25 & $16.6 \pm 1.2$ & $7 / 18$ & $40.4 \pm 3.1$ & $115.4 \pm 14.0$ & $118.1 \pm 10.6$ & NA & NA & NA/NA \\
\hline & & 639 & & & & & & & & & & \\
\hline O'Brien & Australia & $\begin{array}{l}\text { ACTRN12 } \\
611000279\end{array}$ & Surgery & 31 & $53.6 \pm 6.2$ & $5 / 26$ & $33.6 \pm 1.9$ & $94.7 \pm 11.2$ & $103.2 \pm 10.1$ & NA & NA & NA/NA \\
\hline & & 921 & Control & 20 & $52.7 \pm 7.7$ & $7 / 13$ & $33.5 \pm 1.5$ & $95.6 \pm 13.4$ & $101.5 \pm 11.1$ & NA & NA & $\mathrm{NA} / \mathrm{NA}$ \\
\hline Parikh & USA & NCT01423 & Surgery & 29 & $46.8 \pm 8.1$ & $6 / 23$ & $32.8 \pm 1.7$ & $81.9 \pm 7.7$ & $106.3 \pm 10.1$ & $25(86.2 \%)$ & NA & $100.0 \% / \mathrm{NA}$ \\
\hline $2014[25]$ & USA & 877 & Control & 28 & $53.9 \pm 8.4$ & $6 / 22$ & $32.4 \pm 1.8$ & $83.7 \pm 10.8$ & $106.7 \pm 7.7$ & $25(89.3 \%)$ & NA & $100.0 \% / \mathrm{NA}$ \\
\hline Reis & Brazil & NA & Surgery & 10 & $36.7 \pm 11.5$ & $10 / 0$ & $55.7 \pm 7.8$ & $168.6 \pm 28.2$ & NA & NA & NA & NA/NA \\
\hline $2009[26]$ & Brazil & NA & Control & 10 & $42.2 \pm 11.0$ & $10 / 0$ & $54.0 \pm 6.1$ & $160.4 \pm 20.1$ & NA & NA & NA & NA/NA \\
\hline SLIMM-T2D & USA & NCT01073 & Surgery & 37 & $50.7 \pm 10.2$ & $15 / 22$ & $36.2 \pm 3.2$ & $105.7 \pm 13.1$ & NA & $25(67.6 \%)$ & NA & $100.0 \% / 10.5 y$ \\
\hline$[27,28]$ & USA & 020 & Control & 41 & $52.0 \pm 6.2$ & $22 / 19$ & $36.6 \pm 3.8$ & $107.5 \pm 17.8$ & NA & $23(56.1 \%)$ & NA & $100.0 \% / 9.2 \mathrm{y}$ \\
\hline STAMPEDE & USA & NCT00432 & Surgery & 100 & $48.1 \pm 8.2$ & $32 / 68$ & $36.6 \pm 3.6$ & $103.8 \pm 15.8$ & $115.2 \pm 9.8$ & $73(73.0 \%)$ & $65(65.0 \%)$ & $100.0 \% / 8.4 \mathrm{y}$ \\
\hline [29-34] & USA & 809 & Control & 50 & $49.7 \pm 7.4$ & $19 / 31$ & $36.8 \pm 3.0$ & $106.5 \pm 14.7$ & $114.5 \pm 9.4$ & $37(74.0 \%)$ & $26(52.0 \%)$ & $100.0 \% / 8.9 y$ \\
\hline TRAMOMT & Snin & EudraCT & Surgery & 37 & $44.1 \pm 9.8$ & $11 / 26$ & $49.2 \pm 5.9$ & $132.8 \pm 24.4$ & NA & $35(94.6 \%)$ & NA & $24.3 \% / \mathrm{NA}$ \\
\hline ANA[35] & Spatit & $37-24$ & Control & 106 & $47.4 \pm 11.0$ & $34 / 72$ & $46.2 \pm 4.8$ & $123.8 \pm 19.2$ & NA & $103(97.2 \%)$ & NA & $24.5 \% / \mathrm{NA}$ \\
\hline TRIABETES & USA & NCT01047 & Surgery & 46 & $46.8 \pm 7.0$ & $9 / 37$ & $35.5 \pm 3.0$ & $99.7 \pm 13.3$ & $112.7 \pm 10.2$ & $35(76.1 \%)$ & $25(54.3 \%)$ & $100.0 \% / 6.8 \mathrm{y}$ \\
\hline$[36,37]$ & & 735 & Control & 23 & $48.3 \pm 4.7$ & $4 / 19$ & $35.7 \pm 3.3$ & $102.6 \pm 13.8$ & $111.7 \pm 9.5$ & $19(82.6 \%)$ & $16(69.6 \%)$ & $100.0 \% / 5.7 \mathrm{y}$ \\
\hline
\end{tabular}

Table 2: Revised Jadad's Scale assessment

\begin{tabular}{|c|c|c|c|c|c|}
\hline Trial & Randomization & Allocation concealment & Blindness & Withdrawal & Total \\
\hline DIBASY & 2 & 1 & 0 & 1 & 4 \\
\hline Dixon 2008 & 2 & 1 & 0 & 1 & 4 \\
\hline Dixon 2012 & 2 & 1 & 2 & 1 & 6 \\
\hline DSS & 1 & 0 & 0 & 1 & 2 \\
\hline Heindorff 1997 & 1 & 1 & 1 & 0 & 3 \\
\hline Liang 2013 & 2 & 1 & 1 & 1 & 5 \\
\hline Mingrone 2002 & 1 & 1 & 1 & 0 & 3 \\
\hline O'Brien 2006 & 2 & 2 & 0 & 1 & 5 \\
\hline O'Brien 2010 & 2 & 1 & 0 & 1 & 4 \\
\hline O'Brien 2013 & 1 & 1 & 1 & 1 & 4 \\
\hline Parikh 2014 & 2 & 2 & 1 & 1 & 6 \\
\hline Reis 2009 & 2 & 1 & 1 & 1 & 5 \\
\hline SLIMM-T2D & 2 & 2 & 1 & 1 & 6 \\
\hline STAMPEDE & 2 & 1 & 0 & 1 & 4 \\
\hline TRAMOMTANA & 2 & 1 & 0 & 1 & 4 \\
\hline TRIABETES & 2 & 1 & 1 & 1 & 5 \\
\hline
\end{tabular}


Table 3: Outcomes of weight loss by sensitivity analysis

\begin{tabular}{|c|c|c|c|c|c|c|c|c|c|c|}
\hline \multirow{2}{*}{$P$ value } & \multirow{2}{*}{ Overall } & \multicolumn{3}{|c|}{ Follow-up duration } & \multicolumn{4}{|c|}{ Surgical techniques } & \multicolumn{2}{|c|}{ Levels of obesity } \\
\hline & & 1-year & 2-year & \begin{tabular}{|l}
$\begin{array}{l}\text { Long- } \\
\text { term }\end{array}$ \\
\end{tabular} & SG & RYGB & LAGB & BPD & Nonsevere & Severe \\
\hline Random effects & $<0.00001$ & $<0.00001$ & $<0.00001$ & $<0.00001$ & $<0.00001$ & $<0.00001$ & $<0.00001$ & $<0.00001$ & $<0.00001$ & $<0.00001$ \\
\hline Fixed effects & $<0.00001$ & $<0.00001$ & $<0.00001$ & $<0.00001$ & $<0.00001$ & $<0.00001$ & $<0.00001$ & $<0.00001$ & $<0.00001$ & $<0.00001$ \\
\hline \begin{tabular}{|l|} 
With low- \\
quality
\end{tabular} & $<0.00001$ & $<0.00001$ & $<0.00001$ & $<0.00001$ & $<0.00001$ & $<0.00001$ & $<0.00001$ & $<0.00001$ & $<0.00001$ & $<0.00001$ \\
\hline \begin{tabular}{|l|}
$\begin{array}{l}\text { Without low- } \\
\text { quality }\end{array}$ \\
\end{tabular} & $<0.00001$ & $<0.00001$ & $<0.00001$ & $<0.00001$ & $<0.00001$ & $<0.00001$ & $<0.00001$ & $<0.00001$ & $<0.00001$ & $<0.00001$ \\
\hline $\begin{array}{l}\text { Previous } \\
\text { criteria }\end{array}$ & $<0.00001$ & $<0.00001$ & $<0.00001$ & $<0.00001$ & $<0.00001$ & $<0.00001$ & $<0.00001$ & $<0.00001$ & $<0.00001$ & $<0.00001$ \\
\hline Altered criteria & $<0.00001$ & $<0.00001$ & $<0.00001$ & $<0.00001$ & $<0.00001$ & $<0.00001$ & $<0.0001$ & $<0.00001$ & $<0.00001$ & $<0.00001$ \\
\hline
\end{tabular}

SG: sleeve gastrectomy; RYGB: Roux-en-Y gastric bypass; LAGB: laparoscopic adjustable gastric banding; BPD: biliopancreatic diversion;

Table 4: Revised Jadad's Scale

\begin{tabular}{|l|}
\hline Random sequence production \\
\hline a. Adequate (computer generated random numbers or similar methods) (2 points) \\
\hline b. Unclear (a randomized trial but without description of randomization methods) (1 point) \\
\hline c. Inadequate (an alternative allocation without randomization) (0 point) \\
\hline Allocation concealment \\
\hline a. Adequate (a central institution-controlled allocation) (2 points) \\
\hline b. Unclear (random numerical table or other similar methods) (1 point) \\
\hline c. Inadequate (alternative allocation without adequate concealment) (0 point) \\
\hline Blindness \\
\hline a. Adequate (comparable placebo or similar methods) (2 points) \\
\hline b. Unclear (a blind trial without details statement) (1 point) \\
\hline c. Inadequate (inappropriate blind methods or non-blind trials) ( 0 point) \\
\hline Withdrawal \\
\hline a. Description (a detailed statement about the numbers and reasons of withdrawals) (1 point) \\
\hline b. No description (no statement about the numbers and reasons of withdrawals) $(0$ point) \\
\hline
\end{tabular}

$0.00001)$, Roux-en-Y gastric bypass $(P<0.00001)$, laparoscopic adjustable gastric banding $(P<0.00001)$ and biliopancreatic diversion $(P<0.00001)$ (Figure 3$)$.

\section{Levels of obesity}

Among patients with nonsevere obesity (body mass index 30-35; $P<0.00001$ ) and severe obesity (body mass index $>35 ; P<0.00001)$, metabolic surgery was a more effective tool for losing weight against non-surgical treatments (Figure 4).

\section{Remission of type 2 diabetes mellitus}

\section{Overall}

Our pooled outcomes suggested that surgical patients had a higher rate of diabetic remission than those undergoing non-surgical interventions $(P<0.00001)$ (Supplementary Figure S1).

\section{Follow-up duration}

Patients undergoing bariatric surgery achieved significantly higher rate of diabetic remission compared to recipients of conservative therapeutics, based on pooled 
results of 1-year $(P<0.0001), 2$-year $(P=0.004)$ and long-term follow-up $(P<0.0001)$ (Supplementary Figure S1).

\section{Surgical techniques}

In contrast to conserved managements, there was a much better remission rate of diabetes amid surgical recipients, irrespective of sleeve gastrectomy $(P<0.00001)$, Roux-en-Y gastric bypass $(P<0.00001)$, laparoscopic adjustable gastric banding $(P=0.0008)$ and biliopancreatic diversion $(P=0.001)$ (Supplementary Figure S2).

\section{Levels of obesity}

Concerning the diabetic remission rate, both nonseverely $(P=0.0004)$ and severely obese $(P<0.0001)$ sufferers significantly benefited from surgical remedies, compared with non-surgical patients (Supplementary Figure S3).

\section{Secondary endpoints}

\section{Excessive weight loss}

\section{Overall}

Among obese subjects, our quantitative analysis reported that operative treatments induced higher percentage of excessive weight loss against traditional therapeutics $(P<0.00001)$ (Supplementary Figure S4).

\section{Follow-up duration}

Regardless of 1-year $(P<0.00001), 2$-year $(P<$ $0.00001)$ or long-range follow-up $(P=0.006)$, bariatric surgery was far more efficient to eliminate excessive weight than non-surgical interventions (Supplementary Figure S4).

\section{Surgical techniques}

It was a statistical advantage to surgically reduce the excessive mass of obese enrollees instead of conventional treatments, whichever of sleeve gastrectomy

\section{A}

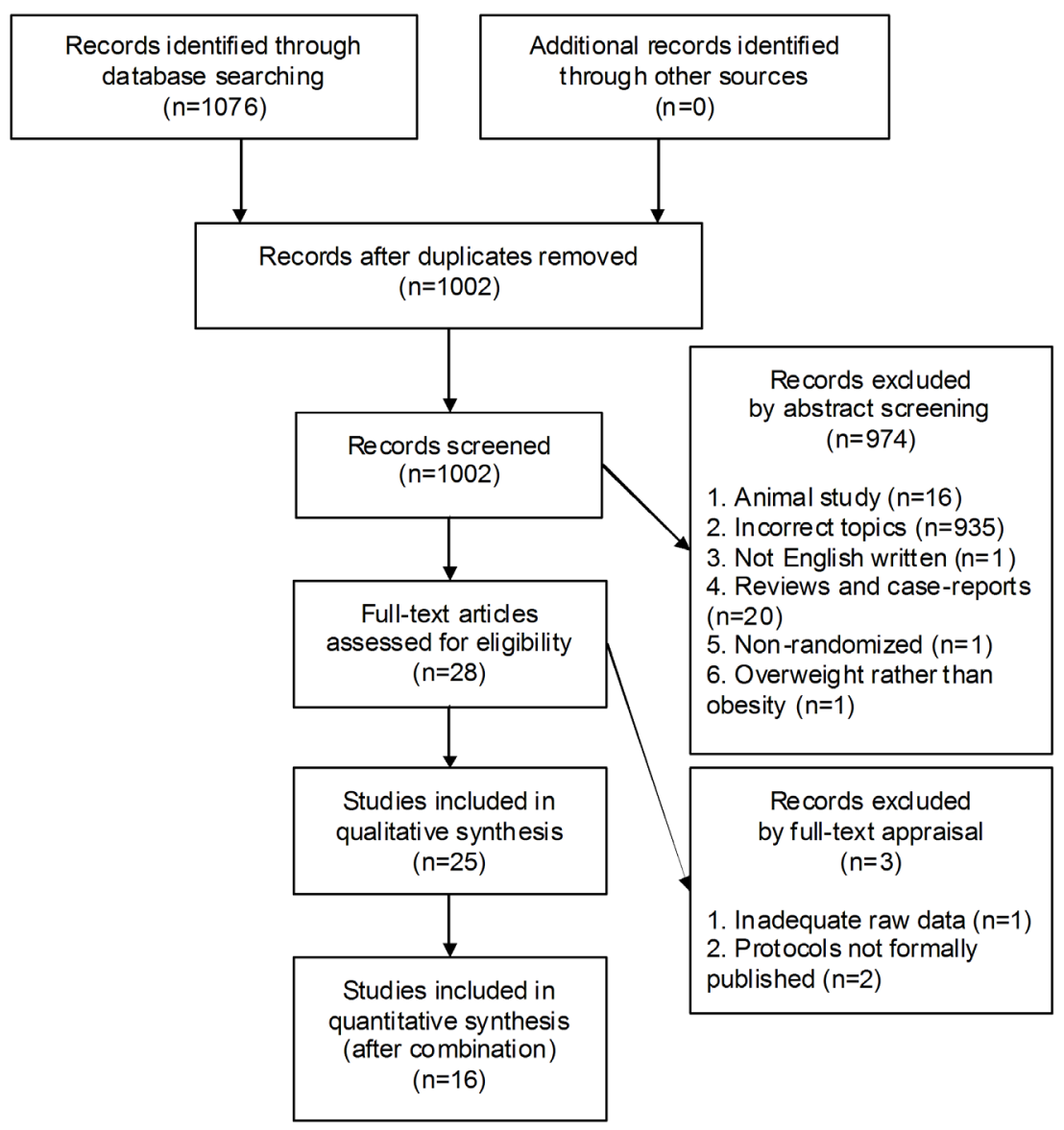

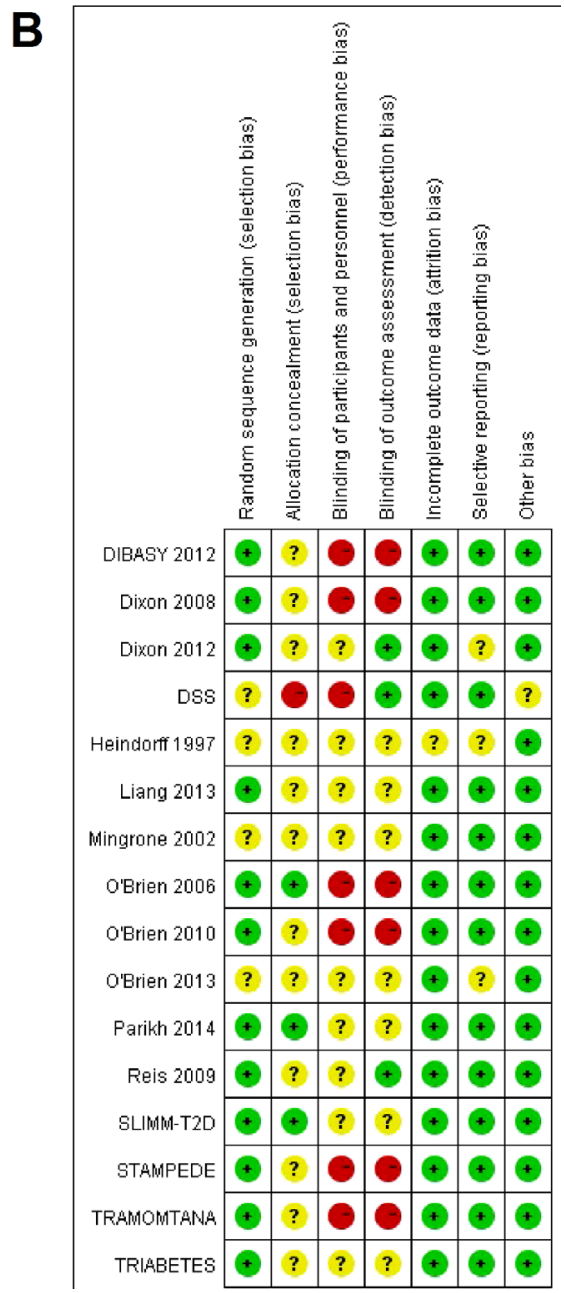

Figure 1: Selection flow chart and risk of bias summary. A. Flow chart of the entire selection process; B. Risk of bias summary. 
$(P<0.00001)$, Roux-en-Y gastric bypass $(P<0.00001)$, laparoscopic adjustable gastric banding $(P=0.0008)$ and biliopancreatic diversion $(P<0.00001)$ was performed (Supplementary Figure S5).

\section{Levels of obesity}

Recipients of bariatric surgery gained greater loss of excessive weight in comparison to those undergoing noninvasive treatments, according to the subgroup analysis of both nonsevere $(P<0.00001)$ and severe obesity $(P<$
0.00001) (Supplementary Figure S6).

\section{Fasting glucose}

\section{Overall}

A better alleviation on fasting glucose was observed among patients undergoing bariatric surgery, in contrast to those receiving conventional remedies $(P<0.00001)$ (Supplementary Figure S7).

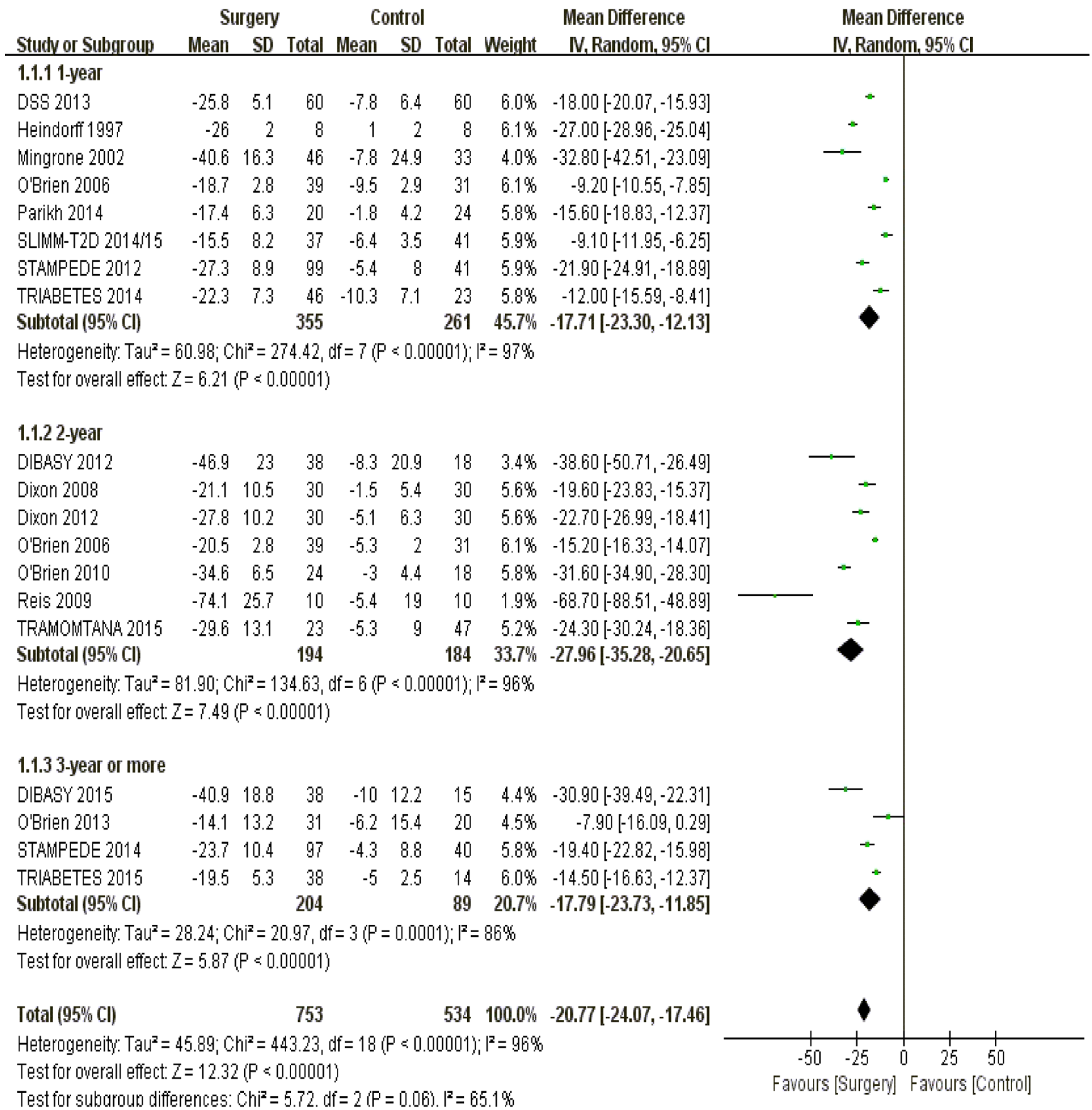

Figure 2: The forest plot of weight loss (kg) in terms of follow-up duration. 


\section{Follow-up duration}

Regardless of 1-year $(P=0.0005), 2$-year $(P=$ $0.0003)$ or long-term follow-up $(P=0.02)$, surgical management was significantly effective in decreasing fasting glucose compared to non-surgical interventions (Supplementary Figure S7).

\section{Surgical techniques}

None of sleeve gastrectomy $(P=0.0005)$, Rouxen-Y gastric bypass $(P<0.00001)$ and laparoscopic adjustable gastric banding $(P<0.0001)$ fell behind in fasting glucose reduction against conserved therapeutics, except for biliopancreatic diversion, which was statistically equivalent to contrastive group $(P=0.05)$ (Supplementary Figure S8).

\section{Levels of obesity}

Compared to non-surgical regimen, both nonseverely $(P=0.001)$ and severely obese $(P<$ $0.00001)$ patients obtained greater downregulation on fasting glucose following the surgical interventions (Supplementary Figure S9).

\section{Glycated hemoglobin}

\section{Overall}

Metabolic surgery led to more reduction on percentage of glycated hemoglobin among obese participants than conventional managements $(P<0.00001)$ (Supplementary Figure S10).

\section{Follow-up duration}

A higher percentage reduction on glycated hemoglobin was surgically achieved among patients with 1-year $(P<0.00001)$, 2-year $(P=0.02)$ and longterm follow-up $(P<0.00001)$, rather than those with conservative interventions (Supplementary Figure S10).

\section{Surgical techniques}

Irrespective of sleeve gastrectomy $(P<0.0001)$, Roux-en-Y gastric bypass $(P<0.00001)$, laparoscopic adjustable gastric banding $(P=0.002)$ and biliopancreatic diversion $(P=0.03)$, bariatric surgery was significantly superior to non-surgical approaches in terms of reducing elevated glycated hemoglobin (Supplementary Figure S11).

\section{Levels of obesity}

There was a greater decrease on glycated hemoglobin percentage following bariatric operations than non-surgical strategies, among nonseverely $(P<0.00001)$ and severely obese patients $(P<0.00001)$ (Supplementary Figure S12).

\section{Waist circumference}

\section{Overall}

Compared to baseline values, a greater loss on waist circumference was obtained amid surgical patients, instead of non-surgical counterparts $(P<0.00001)$ (Supplementary Figure S13).

\section{Follow-up duration}

Among the three subgroups of 1 -year $(P<0.00001)$, 2-year $(P<0.00001)$ and long-term follow-up $(P<$ $0.00001)$, patients that were surgically treated obtained larger decline on waist circumference than those were conventionally cured (Supplementary Figure S13).

\section{Surgical techniques}

Bariatric surgery was more effective to cut down waist circumference than non-surgical interventions, including sleeve gastrectomy $(P<0.0001)$, Roux-en-Y gastric bypass $(P<0.00001)$, laparoscopic adjustable gastric banding $(P<0.00001)$ and biliopancreatic diversion $(P<0.00001)$ (Supplementary Figure S14).

\section{Levels of obesity}

Based on the pooled outcomes featuring nonseverely $(P<0.00001)$ and severely obese sufferers $(P<0.00001)$, more reduction on waist circumference was observed following surgical management in contrast to traditional treatments (Supplementary Figure S15).

\section{Systolic pressure}

\section{Overall}

With respect to decrease on systolic pressure among enrolled participants, metabolic surgery has a significant advantage against non-surgical remedies $(P<0.00001)$ (Supplementary Figure S16).

\section{Follow-up duration}

Regarding the efficacy of systolic pressure reduction, patients with 2-year postoperative follow-up equaled to those receiving conventional interventions $(P=$ $0.30)$, while bariatric surgery was a superior option among sufferers with 1-year $(P=0.001)$ and long-range follow-up $(P=0.02)$ (Supplementary Figure S16).

\section{Surgical techniques}

Bariatric surgery featuring sleeve gastrectomy $(P=$ 0.36), laparoscopic adjustable gastric banding $(P=0.36)$ and biliopancreatic diversion $(P=0.90)$ was statistically comparable with non-surgical strategies in terms of reduction on systolic pressure, except for the dominant efficacy of Roux-en-Y gastric bypass $(P=0.007)$ (Supplementary Figure S17).

\section{Levels of obesity}

In comparison to conservative interventions, bariatric surgery played a preponderant and comparable 


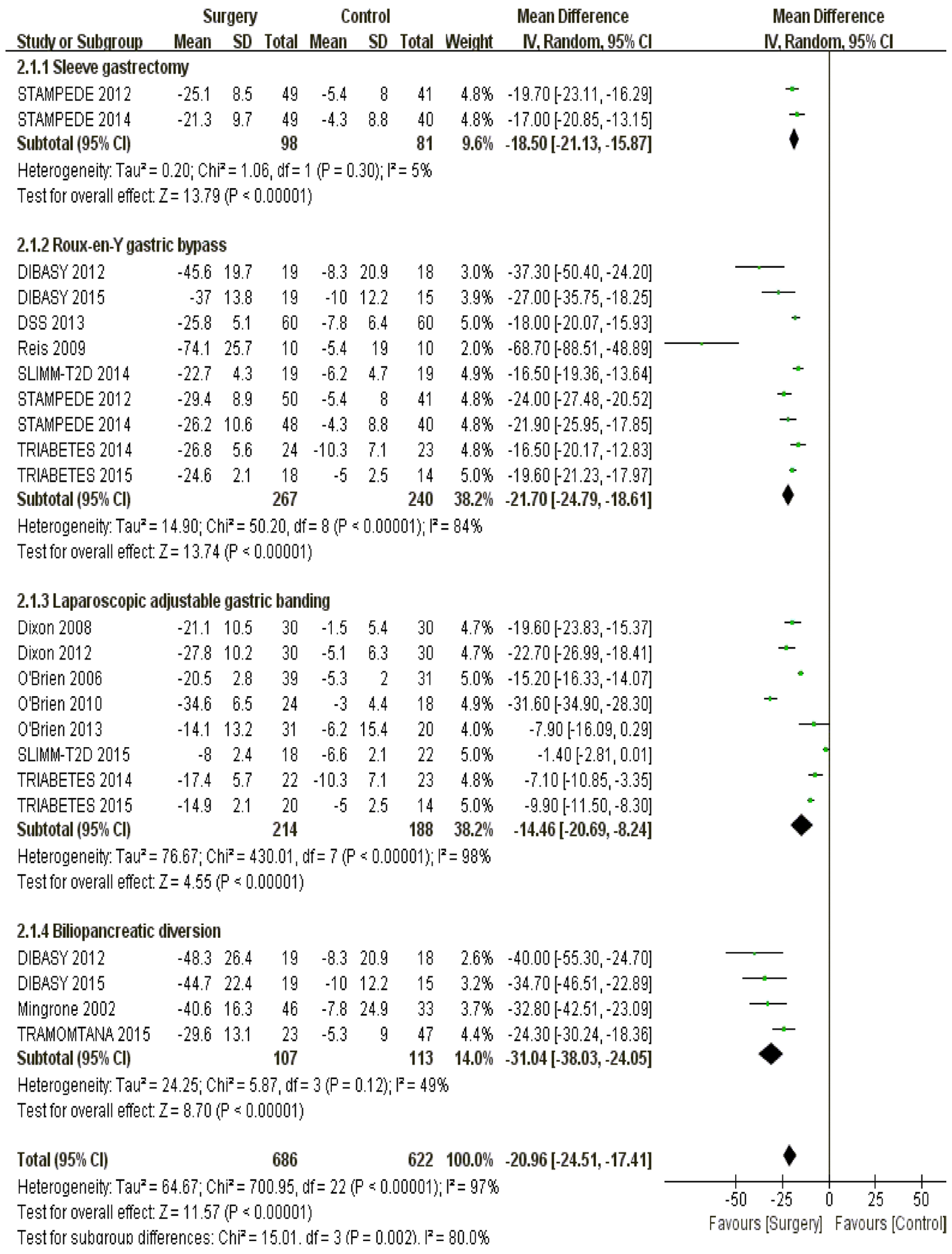

Figure 3: The forest plot of weight loss (kg) in terms of surgical techniques. 
role among nonseverely $(P<0.00001)$ and severely obese patients $(P=0.41)$ respectively, in terms of reduction on systolic pressure (Supplementary Figure S18).

\section{Diastolic pressure}

\section{Overall}

There was no significant difference between surgical and non-surgical strategies towards diastolic pressure reduction among included patients $(P=0.50)$ (Supplementary Figure S19).

\section{Follow-up duration}

Bariatric surgery had a comparable impact on attenuating diastolic pressure against conventional therapies, within patients being followed up for 1-year $(P=0.26)$ and 2 -year $(P=0.55)$. However, surgical interventions were inferior to non-invasive remedies amid patients with long-term follow-up $(P=0.03)$ (Supplementary Figure S19).

\section{Surgical techniques}

Although Roux-en-Y gastric bypass $(P=0.03)$ were in a significantly superior status, the remaining techniques were statistically comparable to conservative regimens concerning the decrease on diastolic pressure, inclusive of sleeve gastrectomy $(P=0.67)$, laparoscopic adjustable gastric banding $(P=0.57)$ and biliopancreatic diversion $(P$ $=0.52)($ Supplementary Figure S20).

\section{Levels of obesity}

Patients undergoing both interventions exhibited comparable efficacy on diastolic pressure reduction

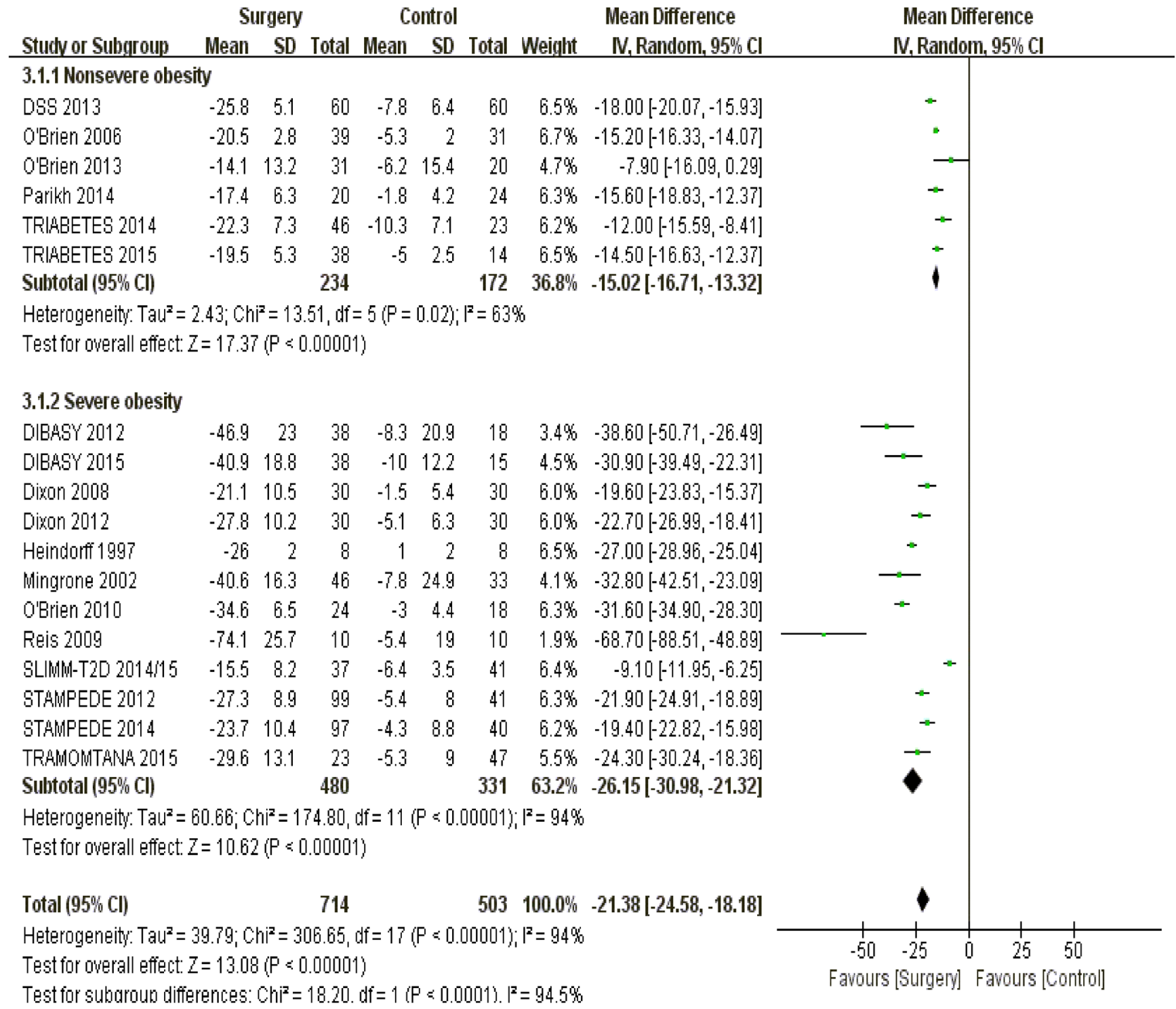

Figure 4: The forest plot of weight loss ( $\mathrm{kg})$ in terms of obesity levels. 
regardless of nonsevere obesity $(P=0.23)$ and severe obesity $(P=0.73)$ (Supplementary Figure S21).

\section{Triglycerides}

\section{Overall}

More decrease on triglycerides level was obtained among surgical participants, other than those receiving conventional therapies $(P<0.00001)$ (Supplementary Figure S22).

\section{Follow-up duration}

According to our pooled outcomes, surgical patients with 1-year $(P<0.0001)$ and 2-year $(P<0.0001)$ followup had a greater decline on triglycerides level than those being conventionally treated, except for the comparable efficacy among patients with long-term follow-up $(P=$ 0.06) (Supplementary Figure S22).

\section{Surgical techniques}

In terms of reducing triglycerides level, Rouxen-Y gastric bypass $(P=0.0005)$ and laparoscopic adjustable gastric banding $(P=0.004)$ exerted a more evident influence among enrolled patients, while sleeve gastrectomy $(P=0.08)$ and biliopancreatic diversion $(P=$ 0.14 ) was therapeutically comparable with conventional remedies (Supplementary Figure S23).

\section{Levels of obesity}

Patients that were surgically managed benefited from a greater loss on triglycerides level than those were non-surgically intervened, regardless of nonsevere obesity $(P<0.00001)$ and severe obesity $(P<0.0001)$ (Supplementary Figure S24).

\section{Total cholesterol}

\section{Overall}

Comparing bariatric surgery and non-surgical interventions, the magnitude of total cholesterol reduction was independent of treatment strategies among obese patients $(P=0.13)$ (Supplementary Figure S25).

\section{Follow-up duration}

Among obese sufferers being followed up for 1-year $(P=0.18)$ and 2-year $(P=0.07)$, there was no therapeutic difference between both interventions. However, patients with long-term follow-up achieved more reduction on total cholesterol following non-surgical managements, in contrast to bariatric surgery $(P=0.03)$ (Supplementary Figure S25).

\section{Surgical techniques}

Besides the preponderant role of biliopancreatic diversion $(P=0.004)$, patients receiving bariatric surgery gained comparable efficacy of total cholesterol reduction against non-surgical enrollees, including sleeve gastrectomy $(P=0.67)$, Roux-en-Y gastric bypass $(P=$ $0.78)$ and laparoscopic adjustable gastric banding $(P=$ 0.62) (Supplementary Figure S26).

\section{Levels of obesity}

Patients with severe obesity featured a surgical advantage in terms of total cholesterol reduction $(P=$ 0.01 ), while nonseverely obese counterparts reported no significant difference between surgical and non-surgical interventions $(P=0.76)$ (Supplementary Figure S27).

\section{High density lipoprotein}

\section{Overall}

A favorable outcome of surgical patients was observed due to their greater increase on high density lipoprotein against those were conservatively healed $(P<$ 0.00001) (Supplementary Figure S28).

\section{Follow-up duration}

Despite of 1-year $(P<0.00001), 2$-year $(P=0.0005)$ and long-term follow-up $(P=0.001)$, metabolic surgery led to more increase on high density lipoprotein among included patients, compared to non-surgical recipients (Supplementary Figure S28).

\section{Surgical techniques}

The recipients of sleeve gastrectomy $(P=0.003)$ and Roux-en-Y gastric bypass $(P<0.00001)$ displayed a higher increase on high density lipoprotein. However, the increase on high density lipoprotein was identically obtained between non-surgical treatments and bariatric surgery of laparoscopic adjustable gastric banding $(P=0.19)$ and biliopancreatic diversion $(P=0.27)$ (Supplementary Figure S29).

\section{Levels of obesity}

Whether participants with nonsevere $(P<0.00001)$ or severe obesity $(P<0.00001)$, bariatric surgery was a more capable pattern of elevating high density lipoprotein than traditional modes (Supplementary Figure S30).

\section{Low density lipoprotein}

\section{Overall}

Among obese patients, both surgical and nonsurgical interventions led to comparable efficacy in reduction of low density lipoprotein $(P=0.21)$ (Supplementary Figure S31).

\section{Follow-up duration}

Comparable to surgical patients with 1-year $(P$ $=0.24)$ and 2-year follow-up $(P=0.14)$, recipients of conventional strategies exceled those with long-term postoperative follow-up duration, with regard to the reduction on low density lipoprotein $(P<0.00001)$ (Supplementary Figure S31). 


\section{Surgical techniques}

Based on subgroup statistics of sleeve gastrectomy $(P=0.34)$, Roux-en-Y gastric bypass $(P=0.71)$ and laparoscopic adjustable gastric banding $(P=0.46)$, it was mathematically comparable between surgical and non-surgical patients regarding the decrease on low density lipoprotein. Nevertheless, patients undergoing biliopancreatic diversion had an advantage over those were conventionally cured $(P<0.00001)$ (Supplementary Figure S32).

\section{Levels of obesity}

There was no statistical significance between bariatric surgery and non-surgical intervention concerning the reduction on low density lipoprotein, according to subgroup analysis of nonseverely $(P=0.76)$ and severely obese patients $(P=0.09)$ (Supplementary Figure S33).

\section{Sensitivity analysis}

Firstly, by interchanging random-effects and fixedeffects models, the overall as well as subgroup outcomes of primary endpoints (weight loss and diabetic remission) were confirmed to be statistically stable (Table 3 ).

Secondly, by eliminating four low-quality trials of DSS, Heindorff 1997, Mingrone 2002 along with O'Brien 2013, the stability of primary endpoints were numerically verified, irrespective of overall or subgroup analysis (Table 3).

Thirdly, since O'Brien 2010 merely contained adolescent participants, the inclusion criteria had been altered by excluding it from pooling analysis. Consequently, results of primary endpoints remained stable under circumstances of novel criteria (Table 3).

Fourthly, by individually removing the eligible studies from primary endpoints, the steadiness of our meta-analysis was graphically proved with aid of STATA 12.0 (Supplementary Figure S34).

\section{Publication bias}

Concerning the primary endpoints of weight loss (Egger: 0.002; Begg: 0.003) and diabetic remission (Egger: 0.001; Begg: 0.043), Egger's test and Begg's test consistently confirmed the presence of publication bias among the included studies (Supplementary Figure S35). However, by "Trim and Fill" method, the pooled outcomes were mathematically equivalent although 2 trials were added for weight loss (before: $P<0.00001$; after: $P<$ 0.0001 ) and 6 added for diabetic remission (before: $P<$ 0.00001; after: $P<0.0001$ ) (Supplementary Figure S36).

\section{DISCUSSION}

Although Golomb et al had retrospectively doubted the dominant role of bariatric surgery at 5-year followup [38], its contrastive efficacy against non-surgical interventions remains advantageous according to our pooled analysis, especially concerning the long-term durability of weight loss and hyperglycemic remission. It is experimentally explanatory that physiological adaptation instead of mechanical reconstruction seems to mainly contribute to the prolonged impacts on energy homeostasis. Adaptive gastrointestinal remodeling, neuroendocrine hormone changes, bile acid signaling and gut microbiota adjustment play combined roles in ameliorating metabolic abnormality and sustaining postoperative energy balance [39]. Nevertheless, along with the increasing follow-up period, the cardiovascular improvement has been therapeutically offset among patients undergoing bariatric surgery, which is confirmed by the comparable outcomes of certain endpoints in our pooled analysis (systolic pressure, diastolic pressure, total cholesterol and low-density lipoprotein). Hence, distant benefits of cardiovascular disorders remain controversial following bariatric surgery, despite Gloy et al [4] had convinced surgeons with short-term advantages on cardiovascular indicators. The close interplay between obesity, diabetes and cardiovascular diseases has been extensively investigated [40]. Following improvements on metabolic status, the lowered risk of cardiovascular accidents is relatively comprehensible among patients with short-term period of follow-up. Thus the long-term insignificance on cardiovascular endpoints seems to blame on those mechanisms independent of ameliorated obesity and hyperglycemia. One possible explanation is that except for biliopancreatic diversion, the prevalent surgical styles including sleeve gastrectomy, Roux-en-Y and laparoscopic adjustable gastric banding fail to adequately impose on cholesterol homeostasis than medication of statins, which consequently culminates in angiosclerosis and hypertension. Besides, cardiovascular dysfunctions are easier to be emotionally and genetically affected, which is inappropriate to simply identify it as metabolic comorbidities [41, 42].

It is empirically regarded that compared to severe obesity, patients suffering from nonsevere obesity manifest with better general conditions which makes it more economical to be conventionally cured. However, deriving from our pooled evidence, nonseverely obese patients should rather receive bariatric surgery than conservative managements, which is similar to those featuring morbid obesity and is a vital addition to current literatures.

Adolescent obesity and overweight crowds have been targeted as further research highlights of bariatric surgery. Uncontrollable weight increase has severer impacts on pubescents than adults, simultaneously damaging their sexual development and intelligence growth [43]. O'Brien et al [23] had reported a randomized trial constituted by juveniles that laparoscopic adjustable gastric banding led to more reduction on excess weight 
than lifestyle intervention, as well as improved life-quality. However, more persuasive investigations are still needed to clarify its clinical worthiness on teenager obesity. Additionally, a substantial proportion of diabetic patients are just overweight instead of clinically obese. But the actual role of bariatric surgery among such populations remains undetermined. By a randomized research, Wentworth et al [44] firstly described a favorable effect of glycemic control and weight loss among overweight patients undergoing laparoscopic adjustable gastric banding in contrast to those with non-surgical treatments. This probably hints that bariatric surgery may fit for all crowds with weight redundancy despite long-term and pooled evidences are scarce.

Notwithstanding the statistical outcomes are of great strength in both methodology and statistics, there are still some limitations within. First of all, the internal heterogeneity is still in a considerable level despite the subgroup analyses have been additionally performed. Difference from chemical examination methods, surgical manipulation techniques and diversified non-surgical interventions (life-style and medications) may contribute to inerasably internal heterogeneity. More accurate grouping on different confounding factors may benefit the source seeking of heterogeneity. Secondly, raw data of life-quality and survival prognostication are inadequate for meta-analyzing, making it less valuable in terms of comparisons on life expectancy. Moreover, mutual comparison of financial burdens is still lacking, blockading a more comprehensive appraisal of both strategies.

Taken together, despite of the shortcomings of surgical therapeutics (a forced alteration of diet habits; large load of exercise required; $8 \%$ of revision rate) [45], bariatric surgery is a more efficient technique for ameliorating obesity and its relevant comorbidities, in contrast to non-surgical interventions. However, more rigorously designed trials with long-term follow-up duration are still required for future supplements and updates.

\section{MATERIALS AND METHODS}

In line with the PRISMA Checklist [46] and Cochrane Collaboration protocols [47], the pooled analysis was designed and implemented in a standard manner. The entire procedures were independently performed by two investigators. Any discrepancy was resolved by mutual discussion.

\section{Search strategy}

In order to guarantee the integrity of literature retrieval, databases of PubMed, Web of Science, EMBASE and Cochrane Library were electronically searched with a search term "bariatric randomized OR bariatric randomised OR obesity surgery". Both abstracts and fulltexts were elaborately examined for fear of omission or ineligible inclusion. Additionally, citation lists of formerly published meta-analysis were screened as well.

\section{Study selection}

Studies were eligibly included because of the following criteria: 1. English-written and officially published trials until December 2015; 2. Randomized controlled trials evaluating the comparative efficacy of surgical versus non-surgical interventions among obese patients (body mass index $>30$ ); 3 . Adequate raw data of interested endpoints including metabolic and cardiovascular indicators;

Studies were eventually eliminated due to the following reasons: 1. Duplicated publications; 2 . Inadequate sample-size $(<10) ; 3$. Lack of follow-up materials;

\section{Data extraction}

With regard to baseline, primary (Weight loss; Remission of type 2 diabetes mellitus) and secondary parameters (Excessive weight loss; Fasting glucose; Glycated hemoglobin; Waist circumference; Systolic pressure; Diastolic pressure; Triglycerides; Total cholesterol; High density lipoprotein; Low density lipoprotein), a well-prepared electronic form was designed to facilitate date extraction from tables, figures, text contents and supplementary information within the eligible trials. Overlapped data deriving from a single registered trial was mathematically combined to prevent repetitive counting. All continuous variables were rounded to one decimal place.

\section{Methodological assessment}

Firstly, a Revised Jadad's Scale [48] was employed in order for a rigorous appraisal of the study design. A total of four categories consisted of the scale, including randomization, allocation concealment, blindness and withdrawal, with a maximum score of seven. Studies graded with four points or more were identified as highquality trials (Table 4).

Secondly, as an addition to Revised Jadad's Scale, Review Manager 5.3 assisted us to summarize the risk of bias amid the qualified literatures. The symbol of green, yellow and red represented low risk of bias, unclear risk of bias and high risk of bias respectively, in terms of seven constituted categories (random sequence generation; allocation concealment; blinding of participants and personnel; blinding of outcome assessment; incomplete outcome data; selective reporting; other bias). The higher 
proportion that green occupies, the lower risk of bias there is [47].

\section{Statistical methods}

Review Manager 5.3 was employed as a statistical platform for our quantitative analysis. The effect-sizes of dichotomous and continuous variable were calculated by models of odds ratio and weighted mean difference respectively, along with $95 \%$ confidence interval. If the source data on endpoints were not offered explicitly, median was statistically regarded as mean while standard deviation was derived from range, interquartile range or $95 \%$ confidence interval as appropriate $[47,49]$. A demandbased merging of subgroups was rigorously conducted to enable sole pairwise comparisons. If necessary, numeric change from baseline values was computed in accord with the statistical instructions of Cochrane Handbook [47]. The overall statistical heterogeneity was quantified by the degree of inconsistency $\left(\mathrm{I}^{2}\right)$ [50]. Revealing a substantially lower heterogeneity, the fixed-effects model was recommended in the setting of $\mathrm{I}^{2}<25 \%$. Otherwise a random-effects model was preferred under the remaining circumstances [51], in order for adjustment of potential variations across the retrieved studies. For the sake of detecting internal stability, sensitivity analysis was accomplished via eliminating low-quality trials, exclusion of controversial studies and comparing the outcome variation between fixed-effects and random-effects models. Moreover, the incorporated outcomes were additionally classified into various subgroups for purpose of more specific and instructive discoveries. With aid of STATA 12.0 , publication bias was numerically examined by Begg's test and Egger's test [52]. A "Trim and Fill" method was conducted in the setting of significant publication bias, in order for adding inadequate studies as well as examining the stability of outcomes. Significant difference was denoted as $P<0.05$.

\section{ACKNOWLEDGMENTS}

We sincerely thank all staff in our department for offering methodological assistances.

\section{CONFLICTS OF INTEREST}

We declare that there is no conflict of interest among all included authors.

\section{GRANT SUPPORT}

This study was financially supported by Research Fund of Public Welfare in Health Industry, Health and Family Plan Committee of China (No.201402015) and National Natural Science Foundation of China (81572413). The recipients are Guobin Wang and Kaixiong Tao respectively.

\section{REFERENCES}

1. Dietz WH, Baur LA, Hall K, Puhl RM, Taveras EM, Uauy R and Kopelman P. Management of obesity: improvement of health-care training and systems for prevention and care. Lancet. 2015; 385:2521-2533.

2. UK NCGC. (2014). Obesity: Identification, Assessment and Management of Overweight and Obesity in Children, Young People and Adults: Partial Update of CG43. (London: National Institute for Health and Care Excellence (UK)).

3. Stegenga $\mathrm{H}$, Haines $\mathrm{A}$, Jones $\mathrm{K}$ and Wilding $\mathrm{J}$. Identification, assessment, and management of overweight and obesity: summary of updated NICE guidance. BMJ. 2014; 349:g6608.

4. Gloy VL, Briel M, Bhatt DL, Kashyap SR, Schauer PR, Mingrone G, Bucher HC and Nordmann AJ. Bariatric surgery versus non-surgical treatment for obesity: a systematic review and meta-analysis of randomised controlled trials. BMJ. 2013; 347:f5934.

5. Yanovski SZ and Yanovski JA. Long-term drug treatment for obesity: a systematic and clinical review. JAMA. 2014; 311:74-86.

6. SAGES guideline for clinical application of laparoscopic bariatric surgery. Surg Obes Relat Dis. 2009; 5:387-405.

7. UK CFPH and UK NCCF. (2006). Obesity: The Prevention, Identification, Assessment and Management of Overweight and Obesity in Adults and Children. (London: National Institute for Health and Clinical Excellence (UK)).

8. Muller-Stich BP, Senft JD, Warschkow R, Kenngott HG, Billeter AT, Vit G, Helfert S, Diener MK, Fischer L, Buchler MW and Nawroth PP. Surgical versus medical treatment of type 2 diabetes mellitus in nonseverely obese patients: a systematic review and meta-analysis. Ann Surg. 2015; 261:421-429.

9. Wing RR, Bolin P, Brancati FL, Bray GA, Clark JM, Coday M, Crow RS, Curtis JM, Egan CM, Espeland MA, Evans M, Foreyt JP, Ghazarian S, et al. Cardiovascular effects of intensive lifestyle intervention in type 2 diabetes. $\mathrm{N}$ Engl J Med. 2013; 369:145-154.

10. Pories WJ, Swanson MS, MacDonald KG, Long SB, Morris PG, Brown BM, Barakat HA, DeRamon RA, Israel G, Dolezal JM. Who would have thought it? An operation proves to be the most effective therapy for adult-onset diabetes mellitus. Ann Surg. 1995; 222:339-350, 350-352.

11. Sjostrom L, Lindroos AK, Peltonen M, Torgerson J, Bouchard C, Carlsson B, Dahlgren S, Larsson B, Narbro K, Sjostrom CD, Sullivan M and Wedel H. Lifestyle, diabetes, and cardiovascular risk factors 10 years after bariatric surgery. N Engl J Med. 2004; 351:2683-2693. 
12. Adams TD, Gress RE, Smith SC, Halverson RC, Simper SC, Rosamond WD, Lamonte MJ, Stroup AM and Hunt SC. Long-term mortality after gastric bypass surgery. N Engl J Med. 2007; 357:753-761.

13. Mingrone G, Panunzi S, De Gaetano A, Guidone C, Iaconelli A, Nanni G, Castagneto M, Bornstein S and Rubino F. Bariatric-metabolic surgery versus conventional medical treatment in obese patients with type 2 diabetes: 5 year follow-up of an open-label, single-centre, randomised controlled trial. Lancet. 2015; 386:964-973.

14. Mingrone G, Panunzi S, De Gaetano A, Guidone C, Iaconelli A, Leccesi L, Nanni G, Pomp A, Castagneto M, Ghirlanda G and Rubino F. Bariatric surgery versus conventional medical therapy for type 2 diabetes. N Engl J Med. 2012; 366:1577-1585.

15. Dixon JB, O'Brien PE, Playfair J, Chapman L, Schachter LM, Skinner S, Proietto J, Bailey M and Anderson M. Adjustable gastric banding and conventional therapy for type 2 diabetes: a randomized controlled trial. JAMA. 2008; 299:316-323.

16. Dixon JB, Schachter LM, O’Brien PE, Jones K, Grima M, Lambert G, Brown W, Bailey M and Naughton MT. Surgical vs conventional therapy for weight loss treatment of obstructive sleep apnea: a randomized controlled trial. JAMA. 2012; 308:1142-1149.

17. Ikramuddin $\mathrm{S}$, Korner J, Lee WJ, Connett JE, Inabnet WB, Billington CJ, Thomas AJ, Leslie DB, Chong K, Jeffery RW, Ahmed L, Vella A, Chuang LM, et al. Rouxen-Y gastric bypass vs intensive medical management for the control of type 2 diabetes, hypertension, and hyperlipidemia: the Diabetes Surgery Study randomized clinical trial. JAMA. 2013; 309:2240-2249.

18. Nguyen KT, Billington CJ, Vella A, Wang Q, Ahmed L, Bantle JP, Bessler M, Connett JE, Inabnet WB, Thomas A, Ikramuddin S and Korner J. Preserved Insulin Secretory Capacity and Weight Loss Are the Predominant Predictors of Glycemic Control in Patients With Type 2 Diabetes Randomized to Roux-en-Y Gastric Bypass. Diabetes. 2015; 64:3104-3110.

19. Heindorff H, Hougaard K and Larsen PN. Laparoscopic adjustable gastric band increases weight loss compared to dietary treatment: a randomized study. Obes Surg. 1997; 7.

20. Liang Z, Wu Q, Chen B, Yu P, Zhao H and Ouyang X. Effect of laparoscopic Roux-en-Y gastric bypass surgery on type 2 diabetes mellitus with hypertension: a randomized controlled trial. Diabetes Res Clin Pract. 2013; 101:50-56.

21. Mingrone G, Greco AV, Giancaterini A, Scarfone A, Castagneto $\mathrm{M}$ and Pugeat M. Sex hormone-binding globulin levels and cardiovascular risk factors in morbidly obese subjects before and after weight reduction induced by diet or malabsorptive surgery. Atherosclerosis. 2002; 161:455462.

22. O'Brien PE, Dixon JB, Laurie C, Skinner S, Proietto J, McNeil J, Strauss B, Marks S, Schachter L, Chapman L and Anderson M. Treatment of mild to moderate obesity with laparoscopic adjustable gastric banding or an intensive medical program: a randomized trial. Ann Intern Med. 2006; 144:625-633.

23. O'Brien PE, Sawyer SM, Laurie C, Brown WA, Skinner S, Veit F, Paul E, Burton PR, McGrice M, Anderson M and Dixon JB. Laparoscopic adjustable gastric banding in severely obese adolescents: a randomized trial. JAMA. 2010; 303:519-526.

24. O'Brien PE, Brennan L, Laurie C and Brown W. Intensive medical weight loss or laparoscopic adjustable gastric banding in the treatment of mild to moderate obesity: longterm follow-up of a prospective randomised trial. Obes Surg. 2013; 23:1345-1353.

25. Parikh M, Chung M, Sheth S, McMacken M, Zahra T, Saunders JK, Ude-Welcome A, Dunn V, Ogedegbe G, Schmidt AM and Pachter HL. Randomized pilot trial of bariatric surgery versus intensive medical weight management on diabetes remission in type 2 diabetic patients who do NOT meet NIH criteria for surgery and the role of soluble RAGE as a novel biomarker of success. Ann Surg. 2014; 260:617-622, 622-624.

26. Reis LO, Favaro WJ, Barreiro GC, de Oliveira LC, Chaim EA, Fregonesi A and Ferreira U. Erectile dysfunction and hormonal imbalance in morbidly obese male is reversed after gastric bypass surgery: a prospective randomized controlled trial. Int J Androl. 2010; 33:736-744.

27. Halperin F, Ding SA, Simonson DC, Panosian J, GoebelFabbri A, Wewalka M, Hamdy O, Abrahamson M, Clancy K, Foster K, Lautz D, Vernon A and Goldfine AB. Rouxen-Y gastric bypass surgery or lifestyle with intensive medical management in patients with type 2 diabetes: feasibility and 1-year results of a randomized clinical trial. JAMA Surg. 2014; 149:716-726.

28. Ding SA, Simonson DC, Wewalka M, Halperin F, Foster K, Goebel-Fabbri A, Hamdy O, Clancy K, Lautz D, Vernon A and Goldfine AB. Adjustable Gastric Band Surgery or Medical Management in Patients With Type 2 Diabetes: A Randomized Clinical Trial. J Clin Endocrinol Metab. 2015; 100:2546-2556.

29. Schauer PR, Kashyap SR, Wolski K, Brethauer SA, Kirwan JP, Pothier CE, Thomas S, Abood B, Nissen SE and Bhatt DL. Bariatric surgery versus intensive medical therapy in obese patients with diabetes. N Engl J Med. 2012; 366:1567-1576.

30. Kashyap SR, Bhatt DL, Wolski K, Watanabe RM, AbdulGhani M, Abood B, Pothier CE, Brethauer S, Nissen S, Gupta M, Kirwan JP and Schauer PR. Metabolic effects of bariatric surgery in patients with moderate obesity and type 2 diabetes: analysis of a randomized control trial comparing surgery with intensive medical treatment. Diabetes Care. 2013; 36:2175-2182.

31. Schauer PR, Bhatt DL, Kirwan JP, Wolski K, Brethauer SA, Navaneethan SD, Aminian A, Pothier CE, Kim ES, Nissen SE and Kashyap SR. Bariatric surgery versus intensive medical therapy for diabetes-3-year outcomes. N Engl J 
Med. 2014; 370:2002-2013.

32. Malin SK, Samat A, Wolski K, Abood B, Pothier CE, Bhatt DL, Nissen S, Brethauer SA, Schauer PR, Kirwan JP and Kashyap SR. Improved acylated ghrelin suppression at 2 years in obese patients with type 2 diabetes: effects of bariatric surgery vs standard medical therapy. Int J Obes (Lond). 2014; 38:364-370.

33. Singh RP, Gans R, Kashyap SR, Bedi R, Wolski K, Brethauer SA, Nissen SE, Bhatt DL and Schauer P. Effect of bariatric surgery versus intensive medical management on diabetic ophthalmic outcomes. Diabetes Care. 2015; 38:e32-e33.

34. Maghrabi AH, Wolski K, Abood B, Licata A, Pothier C, Bhatt DL, Nissen S, Brethauer SA, Kirwan JP, Schauer PR and Kashyap SR. Two-year outcomes on bone density and fracture incidence in patients with T2DM randomized to bariatric surgery versus intensive medical therapy. Obesity (Silver Spring). 2015; 23:2344-2348.

35. Burguera B, Jesus TJ, Escudero AJ, Alos M, Pagan A, Cortes B, Gonzalez XF and Soriano JB. An Intensive Lifestyle Intervention Is an Effective Treatment of Morbid Obesity: The TRAMOMTANA Study-A Two-Year Randomized Controlled Clinical Trial. Int J Endocrinol. 2015; 2015:194696.

36. Courcoulas AP, Goodpaster BH, Eagleton JK, Belle SH, Kalarchian MA, Lang W, Toledo FG and Jakicic JM. Surgical vs medical treatments for type 2 diabetes mellitus: a randomized clinical trial. JAMA Surg. 2014; 149:707715.

37. Courcoulas AP, Belle SH, Neiberg RH, Pierson SK, Eagleton JK, Kalarchian MA, DeLany JP, Lang W and Jakicic JM. Three-Year Outcomes of Bariatric Surgery vs Lifestyle Intervention for Type 2 Diabetes Mellitus Treatment: A Randomized Clinical Trial. JAMA Surg. 2015; 150:931-940.

38. Golomb I, Ben DM, Glass A, Kolitz T and Keidar A. Long-term Metabolic Effects of Laparoscopic Sleeve Gastrectomy. JAMA Surg. 2015; 150:1051-1057.

39. Dixon JB, Lambert EA and Lambert GW. Neuroendocrine adaptations to bariatric surgery. Mol Cell Endocrinol. 2015; 418 Pt 2:143-152.

40. Mozaffarian D. Dietary and Policy Priorities for Cardiovascular Disease, Diabetes, and Obesity: A Comprehensive Review. Circulation. 2016;133:187-225.
41. Dicker D, Yahalom R, Comaneshter DS and Vinker S. Long-Term Outcomes of Three Types of Bariatric Surgery on Obesity and Type 2 Diabetes Control and Remission. Obes Surg. 2015. Dec 30. [Epub ahead of print]

42. Sjostrom L, Lindroos AK, Peltonen M, Torgerson J, Bouchard C, Carlsson B, Dahlgren S, Larsson B, Narbro K, Sjostrom CD, Sullivan M and Wedel H. Lifestyle, diabetes, and cardiovascular risk factors 10 years after bariatric surgery. N Engl J Med. 2004; 351:2683-2693.

43. Dhaliwal J, Nosworthy NM, Holt NL, Zwaigenbaum L, Avis JL, Rasquinha A and Ball GD. Attrition and the management of pediatric obesity: an integrative review. Child Obes. 2014; 10:461-473.

44. Wentworth JM, Playfair J, Laurie C, Ritchie ME, Brown WA, Burton P, Shaw JE and O'Brien PE. Multidisciplinary diabetes care with and without bariatric surgery in overweight people: a randomised controlled trial. Lancet Diabetes Endocrinol. 2014; 2:545-552.

45. Wise J. All obese patients with type 2 diabetes should be assessed for bariatric surgery, says NICE. BMJ. 2014; 349:g7246.

46. Moher D, Liberati A, Tetzlaff J and Altman DG. Preferred reporting items for systematic reviews and meta-analyses: the PRISMA statement. BMJ. 2009; 339:b2535.

47. Higgins J GS. Cochrane Handbook for Systematic Reviews of Interventions Version 5.1.0 [updated March 2011]. The Cochrane Collaboration. 2011.

48. Clark HD, Wells GA, Huet C, McAlister FA, Salmi LR, Fergusson D and Laupacis A. Assessing the quality of randomized trials: reliability of the Jadad scale. Control Clin Trials. 1999; 20:448-452.

49. Hozo SP, Djulbegovic B and Hozo I. Estimating the mean and variance from the median, range, and the size of a sample. BMC Med Res Methodol. 2005; 5:13.

50. Higgins JP and Thompson SG. Quantifying heterogeneity in a meta-analysis. Stat Med 2002; 21:1539-1558.

51. DerSimonian R and Laird N. Meta-analysis in clinical trials. Control Clin Trials. 1986; 7:177-188.

52. Peters JL, Sutton AJ, Jones DR, Abrams KR and Rushton L. Comparison of two methods to detect publication bias in meta-analysis. Jama. 2006; 295:676-680. 